\title{
Ocular Influence of Nano-modified Fullerene Light: 3. Speed and Quality of Visual Information Processing in
}

\section{Man}

\author{
Sergiy A. Gulyar ${ }^{1,2}$, Zynaida A. Tamarova ${ }^{1}$ and Evgeny K. Kirilenko ${ }^{3}$ \\ 1. Department of General Physiology of Nervous System, Bogomoletz Institute of Physiology National Academy of Sciences of
} Ukraine, Kyiv 401601, Ukraine

2. International Medical Innovation Center Zepter, Kyiv 02152, Ukraine

3. "VIRIA”, Kyiv Scientific and Technical Center, Kyiv 03118,Ukraine

\begin{abstract}
Introduction: A new way of converting light radiation is based on the assumption that it is possible for fullerene $\mathrm{C}_{60}$ molecules to influence it. Physical calculations indicate that in this case there are prerequisites for changing the configuration of the quantum-wave structure of the light flux (hyperharmonization). Our previous studies revealed a possibility of obtaining biological responses to the light reorganized by fullerene. We investigated the effect of application of hyperharmonized light through the organ of vision on the neural structures of the brain associated with it. Transformation of the light was created by using glasses with fullerene filters (THE ${ }^{\circledR}$-glasses). The purpose of this work was to establish whether the light that passed through the fullerene-containing filters installed in glasses affects mental performance of a person; in particular, the speed and quality of performing visual-motor tasks similar to those performed by a person using a computer. Methods: We selected eighteen clinically healthy volunteer participants of both sexes aged from 10 to 78 years with computer skills. In the study we used specially made glasses, the filters of which contained $0.33 \%$ of fullerene in polymethylmethacrylate (Tesla Hyperlight Eyewear ${ }^{\circledR}$; THE $^{\circledR}$-glasses), as well as glasses with darkened filters and a frame of similar design (placebo). The spectrum of light that came into the eyes of the participant was natural (intact light, control) or with a weakened blue spectrum, the wave range of which started from $\sim 420 \mathrm{~nm}$ (fullerene light, placebo light). The participants' mental performance was assessed according to the results of specially designed tests: black-white and color tests of digital sorting and the correction test (simple and of increased complexity). Testing was conducted in 3 series: without glasses in natural light (intact light), in glasses with fullerene-containing filters (fullerene light) and in glasses of similar light transmission, but without fullerene (placebo light). The quality control of tests and the calculation of psycho-physiological indicators were performed automatically using the processing program built into the test. The obtained data were processed statistically. Results: It was established that under the action of light passing through filters containing fullerene installed in the glasses, the execution time of the visual-motor task was reduced by $16.5 \%$ (for the black-white digital sorting test), and the number of errors by 2.7 times (in color digital sorting test) compared to testing with intact lighting. Apparently, the fullerene light positively influenced the speed of information processing and attentiveness. A simple correction test with presentation of 2,000 characters showed that when being in $\mathrm{THE}^{\circledR}$-glasses (fullerene light), the fatigue significantly decreased compared to testing with intact light or placebo light. The concentration ratio in fatigue conditions (end of testing) decreased by $16.2 \%$ (fullerene light), by $28.5 \%$ (placebo light) and by $23.6 \%$ (intact light). The speed of information processing and the percentage of concentration in $\mathrm{THE}^{\circledR}$-glasses were higher, and the latent period of the reaction was shorter than with intact or placebo lighting. All this indicates that the light passing through the fullerene filters of $\mathrm{THE}^{\circledR}$-glasses increases the efficiency of information processing. Conclusion: It has been established that the effect of light passing through a fullerene filter on the visual analyzer and the structures of the central nervous system has a positive effect on the mental performance of a person.
\end{abstract}

Key words: Hyperharmonized light, Tesla Hyperlight Eyewear ${ }^{\circledR}$, THE $^{\circledR}$-glasses, fullerene, fullerene-converted light, human mental performance, digital sorting tests (black-white, color), correction tests. 


\section{Introduction}

This is the third article devoted to the consideration of the light role transformed by fullerene in changing the state of the human central nervous system. The first two articles were aimed at studying brain processes according to EEG (electroencephalogram) data.

Mental performance of a person depends on the specificity of the perception of light by the visual analyzer. It is in the retina that receptor cells are located that are sensitive to the action of light and color. The impulses arising in them are transmitted along the optic nerve to different areas of the brain, where the analysis and processing of visual information take place. This process can probably depend on the characteristics of the light effect (spectrum, interruption frequency, power density, polarization). Changes in the configuration of quanta in the light flux, in particular, with the help of fullerene [1] filters, judging by the data we obtained earlier [2,3], can affect the speed and quality of processing visual information by the brain.

The optical properties of fullerene were first considered in the works of D. Koruga [4-6]. Theoretically, the possibility of light radiation being modified by fullerene was predicted. According to the hypothesis of D. Koruga [7, 8], polarized light transmitted through a layer of fullerene molecules changes the arrangement of quanta in the beam, which was called "hyperpolarization" [9]. As a result, such light acquires new properties that can affect biological structures and have a corrective effect in their dysfunctions and possibly enhance and stabilize the physiological response of the photosensitive cells of the eye. Technically, this approach is implemented by creating an optical filter using the method of impregnating of fullerene $0.33 \%$ in polymethyl methacrylate organic glass (PMMA), 2 mm thick [9].

The effect of hyperpolarization can be obtained in

Corresponding author: Sergiy Gulyar, M.D., Ph.D., D.Sc., professor, research fields: medicine (including environmental medicine), physiology, electromagnetic and light therapy (Bioptron, laser, LED). two ways. If the light was prepolarized (for example, in the Bioptron device), then the electromagnetic waves oscillating in one plane are further modulated, resulting in uniformly distributed spiral-ring structures in the flow from light quanta (Tesla toroids). All incoming quanta are converted. Their location in the Tesla toroids corresponds to a configuration close to the spiral biological structures (Fibonacci law). The scheme of light transformation was previously published by us [10]. Due to the resonance arising in tissues having the like structure on which such light falls, there develops a biological response.

The second path (Fig. 1) presents transformation of the diffused light that enters the eyes in a natural way. In this case, part of the quanta from the surface of the fullerene filter is reflected, but those that have penetrated can also be transformed, as described above. This is probably enough to act on the photosensitive structures of the eye. Thus, we also receive light with additional properties ("hyperharmonized"), which provides a biological effect. Considering the physical and biophysical basis of the origin of the light converted by fullerene, it was named "Quantum Hyperlight $^{\circledR}$ ", and the glasses with installed fullerene optics/filters are Tesla Hyperlight Eyewear $^{\circledR}$ (THE ${ }^{\circledR}$-glasses).

The physical side of these phenomena does not yet have a solid explanation, which is natural due to its 3-year-old "youth". We hope for the future effective joint efforts of physicists, biophysicists and biologists. In this article we will continue to consider the biological response to a new light factor for biologists and physicians, creating an effect called hyperpolarization/hyperharmonization.

Our previous experimental animal studies have shown that light transmitted through a fullerene filter has statistically significant biological activity. It is proved that a long-term (over 8 months) coverage of the whole animal with such light not only does not adversely affect the functional state of the animals, but also improves their quality of life. In laboratory animals 


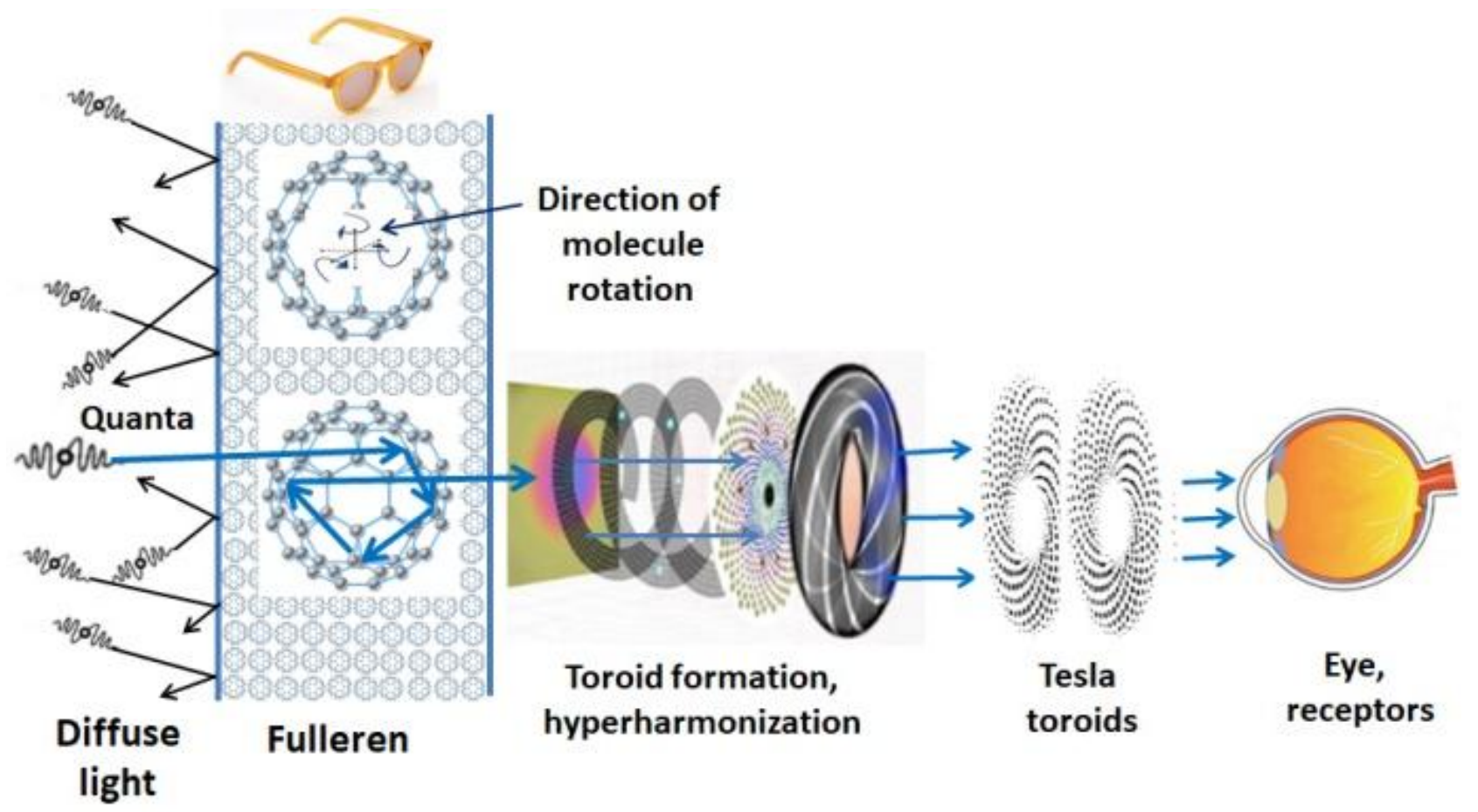

Fig. 1 Scheme of converting the scattered light to hyperharmonized (in accordance with the hypothesis of D. Koruga [8]).

that were under fullerene light, we fixed slowing down of such signs of aging as loss of the body weight and mobility reduction [11].

Assessment of the dynamics of behavioral pain and non-pain reactions to the dosed pain (formalin test) in animals revealed an analgesic effect. It manifested itself in the fact that the application of "carbon" light to the locus of pain/inflammation or to the acupuncture point caused a significant weakening of the pain reaction (analgesic effect) and an increase in the duration of sleep (sedative effect). The sedative effect registered during application of the fullerene light to the acupuncture point significantly exceeded the values obtained under the action of other poly- and monochromatic light ranges [10].

To identify the biological effect of the fullerene light on the human body and assess its value in the ocular path of the light application (THE ${ }^{\circledR}$-glasses), our first focus was on the state of electrical activity of the brain. We obtained significant differences in the delta and theta ranges, which indicated changes in the state of default networks. We revealed reduced EEG activity in the alpha range in the right frontal zone, increased EEG activity in the delta range in the temporal and posterior temporal zones, and increased EEG activity in the theta range in the posterior singular cortex. Totally, these data indicate the development of a more contemplative attitude to the introspective emotional experiences of past events with their active involvement in figurative modeling of a hypothetical future [2, 12]. We also applied an experimental model of the standard situation of "blinding" by oncoming vehicles, in which the driver of a vehicle falls. It is allowed to evaluate the dynamics of sensorimotor reactions and their relationships against the background of EEG recording. We revealed a decrease in the ratio of the latent periods of these reactions when using light modified by fullerene. This indicates an improvement in the rate of inter-hemispheric information processes and an increase in the quality and effectiveness of decisions made. It was proved a significant increase in the activity of neurons located in the posterior singular cortex, in the theta and alpha ranges. This characterizes improved coordination of visual information, attention, 
assessment of the position of one's body, inhibition of irrelevant information, and optimization of decision-making processes. The result of the identified changes was an increase in the speed of central information processing and a shift in focus from image processing to decision-making algorithms [3].

The effectiveness of psychophysiological methods for assessing the influence of the environment on the functional performance of the central nervous system was previously proved by us in studying the action of extreme factors. In particular, it was established during a long stay of a person under conditions of increased pressure of the gas medium in underwater laboratories, pressure chambers or in the water environment. Under these conditions, the nervous structures are exposed to the narcotic action of nitrogen, the toxic action of oxygen, endotoxic effects due to physical overload, decompression disorders, etc. [13-15]. In this case, the search for deviations was quite simple, but difficulties arose in the differential diagnosis of the results obtained. On the other hand, when selecting the same candidates (highly trained with a stable psyche) for work in extreme conditions, there were methodological difficulties in predicting their future sustainability. Therefore, the requirements for tests in the aspect of searching for highly sensitive methods have always been relevant, which required the development of new approaches that ensure maximum objectivity of the results obtained. As a result, an optimal package of techniques was formed, leading among which were correctional. They possessed the above properties, were not burdensome to perform and did not cause negative reactions from the participants.

The psycho-physiological methodological approach has a great inheritance $[16,17]$. We meet a variegated picture of the diversity of tests, the purposes of their application, interpretation of results, technical quality and objectivity, as well as the level of experimenter's training. Therefore, a professional approach to test methods has shown that the choice of reliable options is not so wide. This led us to the need to combine previously proven tests, in particular, our previous studies, with modern computerized base for presenting, executing and evaluating the results without operator participation. In this case, it was provided the maximum sensitivity of the test and objectivity of the result.

Based on the lack of experimental data on the effect of the luminous flux transformed by the fullerene filter on the state of the considered sides of the functioning of the central nervous system, we continue to consider its biological effects. We assume that if the influence of this factor was revealed at the level of brain electrical activity, then at the level of individual psycho-physiological functions such manifestations should also be noticed. The working hypothesis of the present research was that the impact of fullerene light on the visual analyzer in humans can cause a physiological effect, manifested in the change in mental performance, possibly, its optimization. In this study, glasses with the filters described above $\left(\mathrm{THE}^{\circledR}\right.$-glasses) were used. To increase the reliability of the results, we used the placebo method (glasses with darkened filters of similar design and similar in physical characteristics) $[18,19]$. We hoped that in case of a positive effect, there would be grounds for recommendations on the use of fullerene light to optimize a person's daily life (mental performance).

The aim of the work was to establish whether the diffused light transformed by the fullerene, affects the mental performance of a person, in particular, on the speed and quality of performing visual-motor tests of varying degrees of complexity under conditions of moderate and elevated intellectual loads.

\section{Research Methods}

\subsection{Volunteer-Participants}

The study involved 18 clinically healthy volunteer-participants of both sexes aged from 10 to 78 years old with computer skills. Testing was carried out in laboratory rooms of the A. A. Bogomoletz Institute of Physiology, NAS of Ukraine, adapted to the 
conditions of the experiment, in compliance with the Helsinki Declaration, adopted by the General Assembly of the World Medical Association (2010) and with the permission of the Commission on Bioethics of the A. A. Bogomoletz Institute of Physiology, NAS of Ukraine.

When performing tests, a guarding mode was provided in which the subject was not subjected to interference affecting the result: convenient location, no unauthorized persons, telephone calls, etc.

Each of the participants performed tests without glasses (intact light, control), in dark glasses, the filters of which did not contain fullerene (placebo light) and in $\mathrm{THE}^{\circledR}$-glasses, the filters of which contained 0.33 of fullerene (fullerene light). The spectrum of light that came into the participant's eyes was natural (intact light) or with a weakened blue range of the spectrum, the wave range of which started from $420 \mathrm{~nm}$ (fullerene light, placebo light). Examinations with each option of light exposure were conducted separately on different days at the same time.

\subsection{Tests}

The methodological framework is based on keyboard-display technologies [19], taking into account the experience of previous non-computerized test generations $[16,17,19,20]$. They used specially developed tests with different sensitivity to stable and "young" nerve connections and allowing assessing mental performance of a person. A program for automatic processing and documentation of results in real time was built into each test.

2.2.1 Efficiency of the Search Operations (Monochromatic Black-White Tables for Digital Sorting)

The test is based on finding randomly arranged numbers 1-25 as fast as possible. The participant, on the computer monitor screen, finds and places the numbers in the table in the order of their increase. Main indicators are the duration of execution and the number of errors. The test reveals the intelligence, adequacy of the participant and his mental performance when doing simplified tasks. This test allows us to determine the speed of the nervous processes in the central nervous system (CNS) at the moment and assess the functional age of the structures responsible for the performance of this visual-motor task.

For each participant, an individual "plateau" was developed for its main indicators (3-7 presenting's with an interval of more than 5 minutes). Upon the participant's performance on the "plateau" (a consistently repeated test result without glasses), it was suggested to put on the corresponding glasses, and after a 10-minute adaptation the test was performed.

2.2.2 Efficiency of Performing Operations with Attention Switching (Polychromatic Black-Red Tables for Digital Sorting)

The participant on the computer monitor screen should, with the maximum speed, look for and sort in the table, in turn, 24 black numbers in ascending and 25 red numbers in descending order. For each subject, an individual "plateau" was developed for its main indicators (3-7 table presentations with an interval of at least 5 minutes). The main indicators are the duration of execution and the number of errors. This test allows us to assess the speed of switching attention in conditions requiring high concentration and quick response.

2.2.3 Ability and Quality of Mental Load Performance in the Lack of Time Conditions (Correction Test: Simple and Increased Complexity)

The participant is offered a table consisting of 2,000 visual images (E. Landolt rings [21]), oriented randomly in 8 directions. Each ring carries the same amount of information. The test requires from the subject a quick reaction to the appearance on the screen of the ring with which it is necessary to complete the previously ordered task as quickly as possible. At the end of the test, the concentration ratio $(\mathrm{Cr})$ was calculated:

$$
C r=\left(\left(T \_i n f-F \_i n f-S \_ \text {inf }\right) / Z \_i n f\right) \times 100
$$

where $T_{-}$inf is the amount of correctly processed 
information (bit), $F \_$inf is the amount of erroneous information, $S \_$inf is the amount of missing information, $Z$ _inf is the total information volume of the task (test).

The program of processing test results automatically calculates, taking into account the information capacity of the table, machine delay time and tactile response time of a person: the number of correctly and incorrectly processed information, forgetfulness and failures, concentration, speed of information processing as a whole and each correctly processed symbol, average switching time and fixing on one ring, time of switching and fixing attention on rings with different orientations, the total amount of processed information, rate of the task processing, time spent for processing of 1 bit of information. The protocol of work with the table is automatically displayed, the schedule of work with the table, etc. is built.

The correction test was performed in two versions: simple and of high complexity. This made it possible to evaluate not only the reaction rate, attentiveness, etc., but also the degree of fatigue of the participant.

\subsubsection{A Simple Correction Test for Fatigue}

The participant was offered a table consisting of 2,000 Landolt rings. It was necessary to mark the moment of appearance of the character by pressing the "space" key. The task was to cross out a ring with a given orientation with a maximum speed in the presence of a constantly displayed table processing code. In this case, rings with a random time delay appeared successively. When re-working with the table, the arrangement of rings changed randomly. Each participant performed the test without glasses on the first day. The next day, he put on $\mathrm{THE}^{\circledR}$-glasses and after 10 minutes of adaptation, performed the test, on the third day, darkened placebo glasses in the same way.

2.2.3.2 Correction Test for Fatigue of Increased Complexity

The test required the participant to mark the "necessary" rings appearing on the screen with a certain orientation of the gap by the left hand. Along with this, "wrong rings" with a different orientation of the gap appeared on the screen from time to time. These rings should have been marked with the right hand. In total, 2,000 rings were continuously presented, of which the "correct" were about $10 \%$ (the interval between them was random). The scheme of glasses application was similar to the above.

\subsection{Statistical Analysis}

Test execution parameters were estimated automatically using a computer programs. The leading criteria for the quality of the tests were the speed of information processing, the number of errors, the time spent for processing of 1 bit of information, $\%$ of efficiency decrease (fatigue), etc.

For each variable we determined its average value from the entire array of the data obtained. Results are presented as mean \pm SEM. To determine the statistical significance of the differences between the test without glasses, under the influence of fullerene light (THE ${ }^{\circledR}$-glasses) and placebo light we used Student's criterion. The difference between the groups was considered statistically significant at $p<0.05$.

\section{Results}

\subsection{Black-White Digital Sorting Test}

The duration of the test varied in a wide range. For all participants, it decreased with the practice.

As can be seen from Fig. 2, the duration of the test on the first day varied among 5 participants in a wide range from 9.37 to 32.24 s. During repeated and subsequent testing, all participants showed a reduction in the time spent on processing digital information. On the last day, this time ranged from 6.25 to $13.95 \mathrm{~s}$. Thus, the learning process took place. This fact we took into account in further research. Before offering the participant to perform a digital test in fullerene light or placebo light conditions, each of them carried out a learning process. 


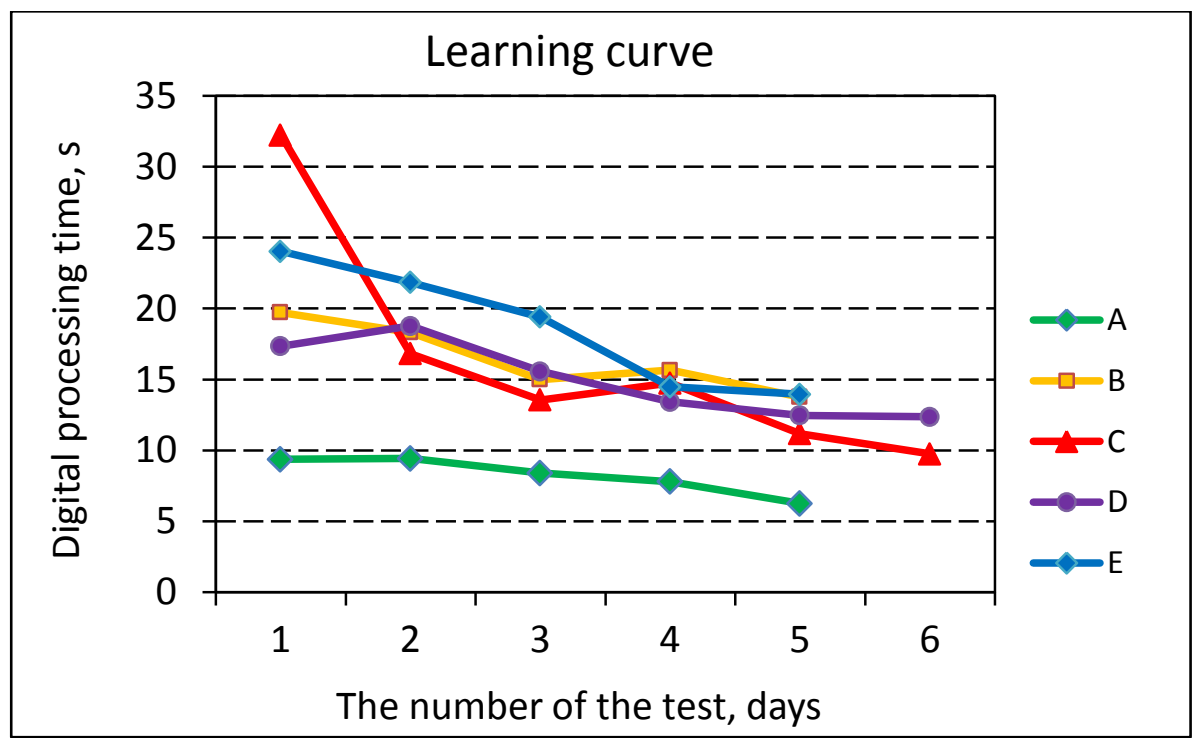

Fig. 2 Change in the time spent on processing digital information during 6 days of the study for 5 participants (A-E). Average values for each day are obtained during 3-7 repeats of a black- white digital test (intact light).

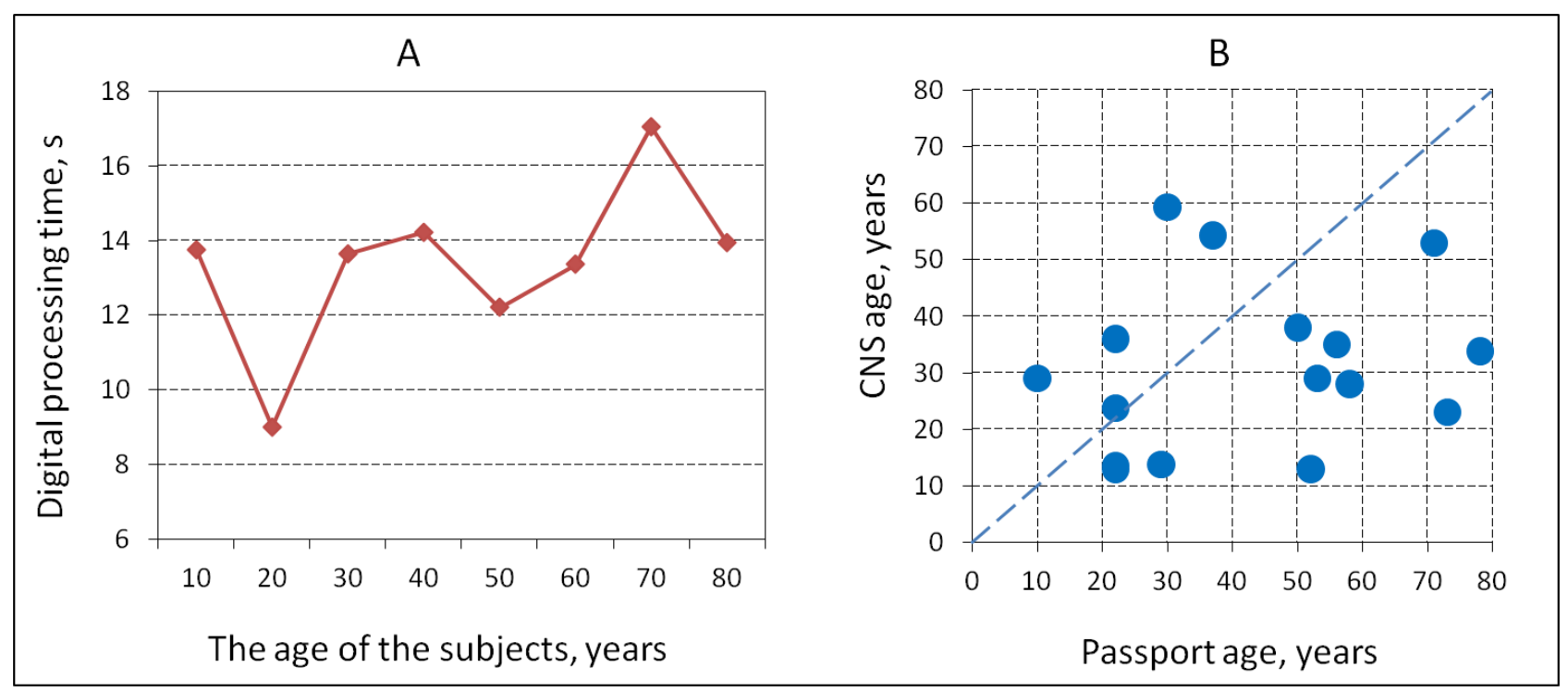

Fig. 3 The average time spent on processing digital information (black- white digital sorting test, intact light) for participants of different ages (A) and the correlation of the age of the CNS structures responsible for performing the visual-motor task with the participants' passport age $(B)$.

In our studies, we had participants of different ages. We tried to assess how age affects the speed of processing digital information. For this purpose, we compared the test time of indicated participants after passing the training period. Fig. 3A shows the average time for each age of participants to complete the digital sorting test. For each participant we took the minimum time to complete the test, then determined the average time for participants in this age group (with an interval of 10 years). Fig. 3 shows that the minimum processing time of digital information (8.99 s) was required for participants aged about 20 years, and the maximum (17.06 s) - about 70 years. In other age categories, this time was kept within 12-14 s.

The age of the structures responsible for performance of the visual-motor task for participants of different passport age (10-78 years) ranged from 13 to 59 years. Correlation between these two parameters was weak. As 


\section{Visual Information Processing in Man}

can be seen from Fig. 3B, for all participants of 50 years and older, the "CNS age" indicator was significantly lower than the passport age, i.e. they used more "young" connections, which are determined by life experience. On the other hand, only 2 participants up to 40 years old showed the "CNS age" below the passport. The rest (5 participants) "CNS age" was higher than the passport age. Obviously, the age of the central nervous system is determined not only by the passport age, but also by life experience and individual human characteristics (type of nervous system, level of intelligence, etc.).

After completing the training period, 16 participants performed a black-white digital sorting test under the influence of all light factors.

From the data obtained it follows that duration of the black- white test of digital sorting with placebo lighting did not significantly differ from the results observed with intact lighting (Fig. 4): $15.6 \mathrm{~s}$ and $15.4 \mathrm{~s}$, respectively ( $101.6 \%$ of the control). In contrast, the light that passed through $\mathrm{THE}^{\circledR}$-glasses shortened the test duration to $12.9 \mathrm{~s}$ ( $83.5 \%$ of the control). The difference with the data obtained in intact and in placebo conditions is statistically significant.

In the group where the test was performed in
$\mathrm{THE}^{\circledR}$-glasses, the age of the CNS structures that participated in performing the black-white digital sorting test averaged 29.6 years ( $85.7 \%$ of the control). The difference with the control (intact light) (34.6 years) is statistically significant. Thus, thanks to the use of light converted by fullerene, "rejuvenation" was achieved by $14.3 \%$. The age of the central nervous system structures under the action of placebo light was not statistically different from the control (Fig. 4): the average group of the central nervous system was 33.9 years old ( $98.0 \%$ of the control), and 34.6 years (100\%) with intact lighting.

Thus, it was found that performing a black-white digital test with an action on a visual analyzer of light converted by a fullerene filter shortens the time it takes to do it by $16.5 \%$ compared to intact light. After application of the fullerene light, the CNS age decreased by $14.3 \%$. On the other hand, the use of placebo light did not significantly change the duration of the black-white digital test and the age of the CNS structures involved in this process, compared with intact light. It was concluded that fullerene-light facilitated the effectiveness of search operations using black-white digital sorting test.

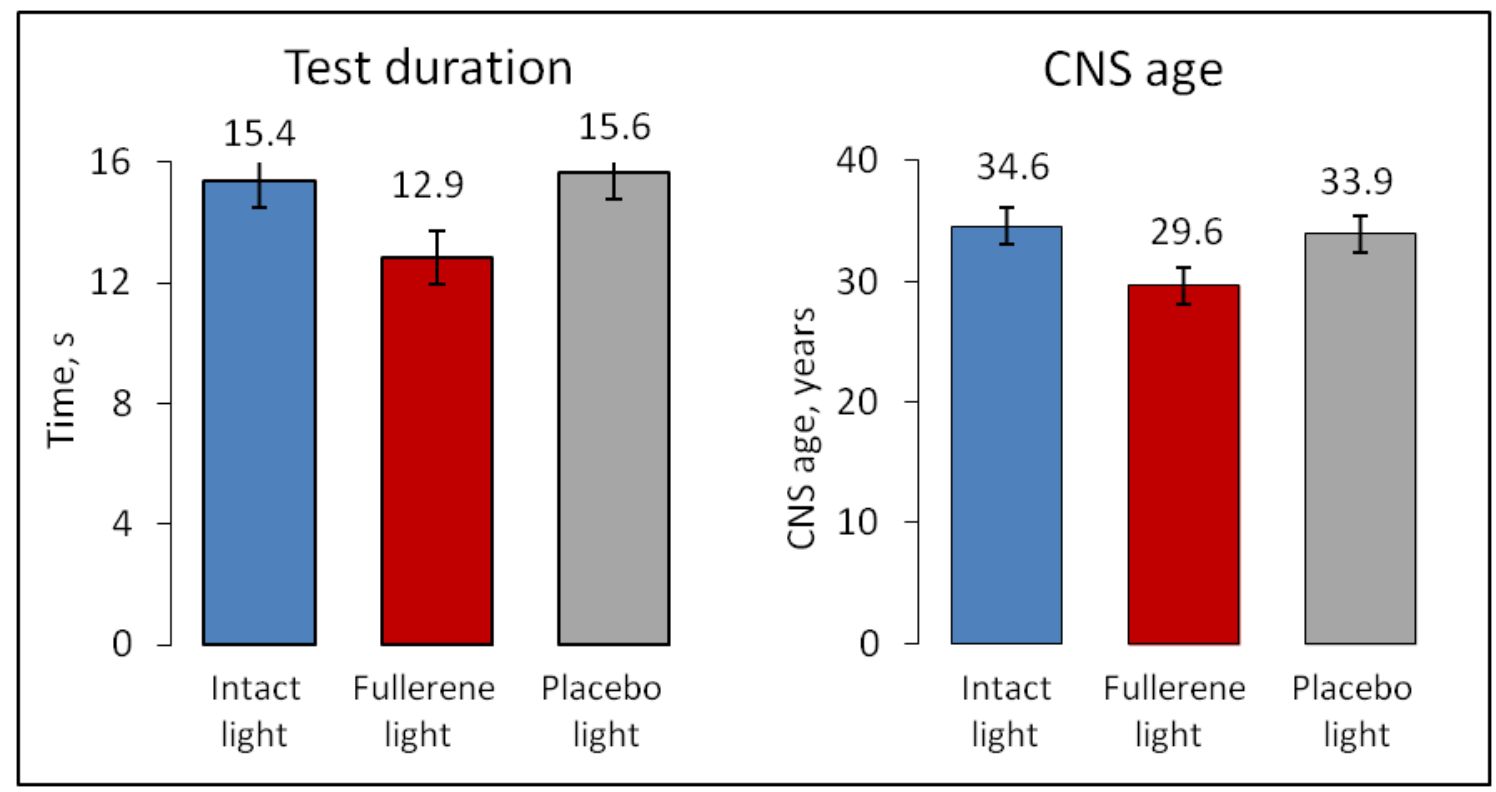

Fig. 4 Duration of the black-white test of digital sorting and the age of the structures of the central nervous system participating in this process under the action of different versions of the light on the eyes. There are given mean values of the group and standard error of the mean. 


\subsection{Color Digital Sorting Test}

The effectiveness of switching operations (black-red digital sorting test) under the influence of fullerene light was studied in 16 participants. This test required increased concentration, good memory, and a quick response for timely attention switching. In this case, the following were evaluated: the duration of the test, the Number of errors and the speed of switching attention are from one character to another.

With repeated testing of the same participant, it was possible to see that the total test time and the time to switch from one character to another were shortened, and the number of errors was significantly reduced. This indicated that the learning process was taking place. We took this fact into account when studying the effectiveness of the considered variants of light. The test under the action of each version of the light was carried out only after each participant passed the training period.

Analysis of these data showed that with the use of fullerene glasses, only one of the 16 participants experienced an increase in the duration of the test and the number of errors compared with intact lighting. For all others fullerene light facilitated fulfillment of the test. Influence of the fullerene light on the group average duration of the color digital test, the number of erroneous points and the speed of switching from one symbol to another is shown in Fig. 5.

As can be seen from Fig. 5, a color digital test under the action of fullerene light was performed in a shorter time than with intact light (105.6 s versus $118.0 \mathrm{~s}$ ). The average time for switching attention from one symbol to another in this case was $1.85 \mathrm{~s}$, and with intact light it was $2.03 \mathrm{~s}$. At the same time, the participants made fewer mistakes than in the case of the test with placebo light and even more so with intact light. The sum of errors in points was 3.06, 4.0 and 8.4, respectively. The use of fullerene light reduced the number of errors by 2.7 times compared to testing with intact light. These data indicated that fullerene-light had a positive effect on attentiveness and thus improved the mental performance of a person.

\subsection{Mental Stress under Time Pressure (Simple Correction Test for Fatigue)}

The data obtained from 10 participants are shown in Figs. 6 and 7. In Fig. 6 it is seen that the latent period of the visually-motor reaction by the end of the test became more variable compared to the reaction to the first displayed characters. It is noticeable that when tested under the action of fullerene light, the scatter was

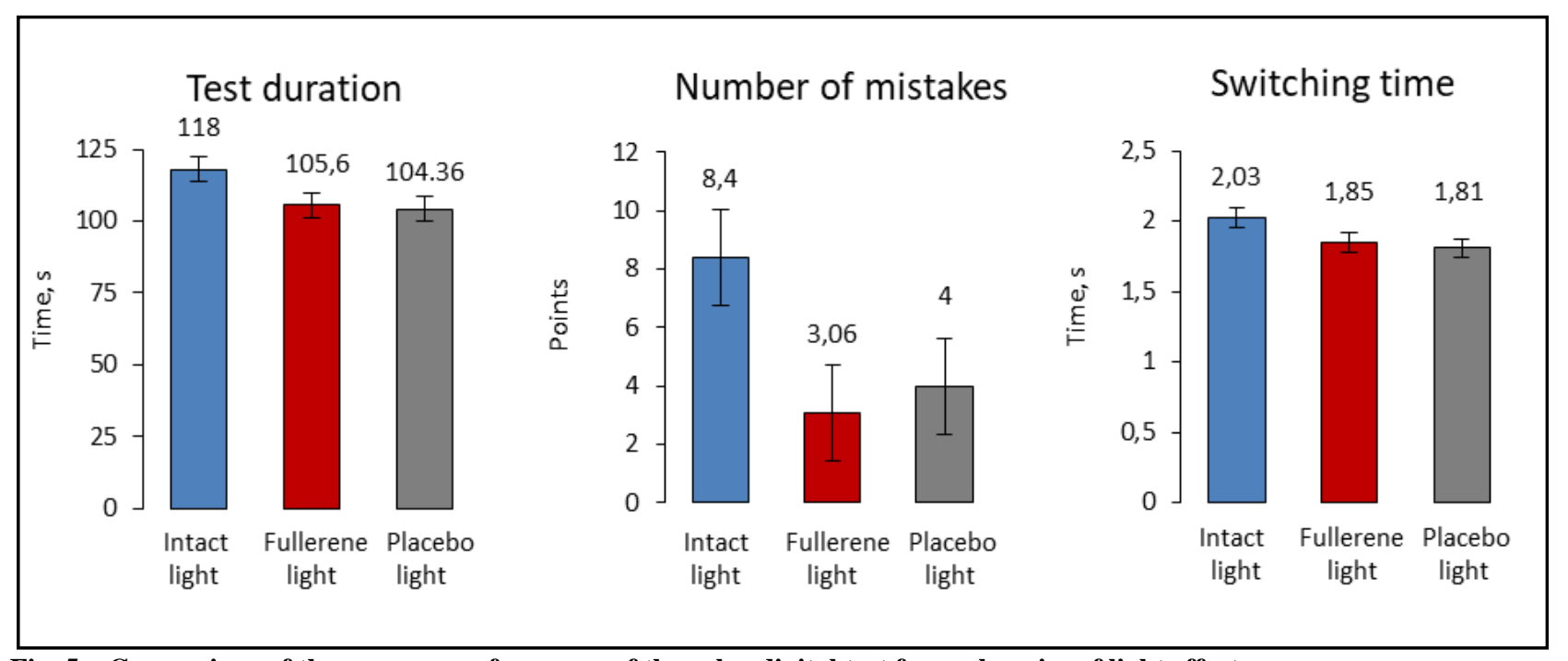

Fig. 5 Comparison of the average performance of the color digital test for each series of light effects. There are indicated mean values and standard errors. 

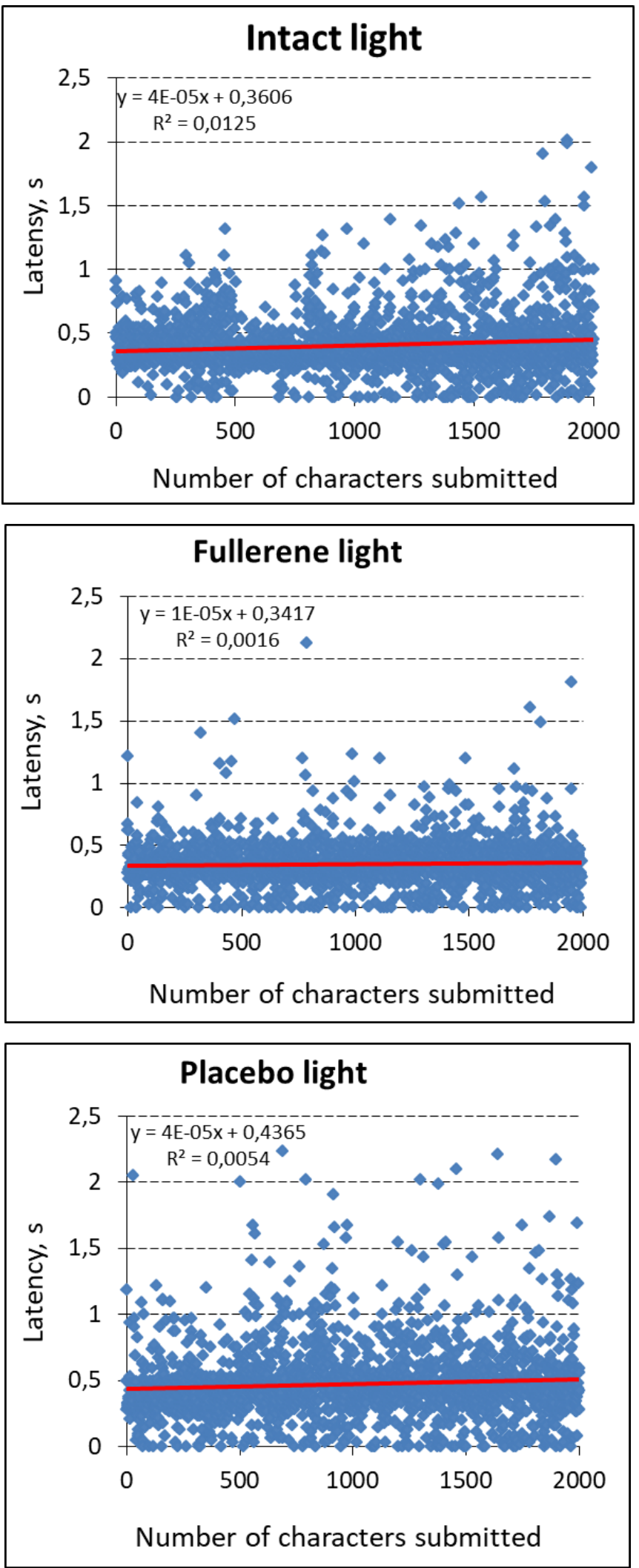

Fig. 6 The latent period of the reaction to the presentation of 2,000 characters from the same participant (direct experimental data) when performing a simple correction test under the influence of the studied variants of lighting the eyes. 

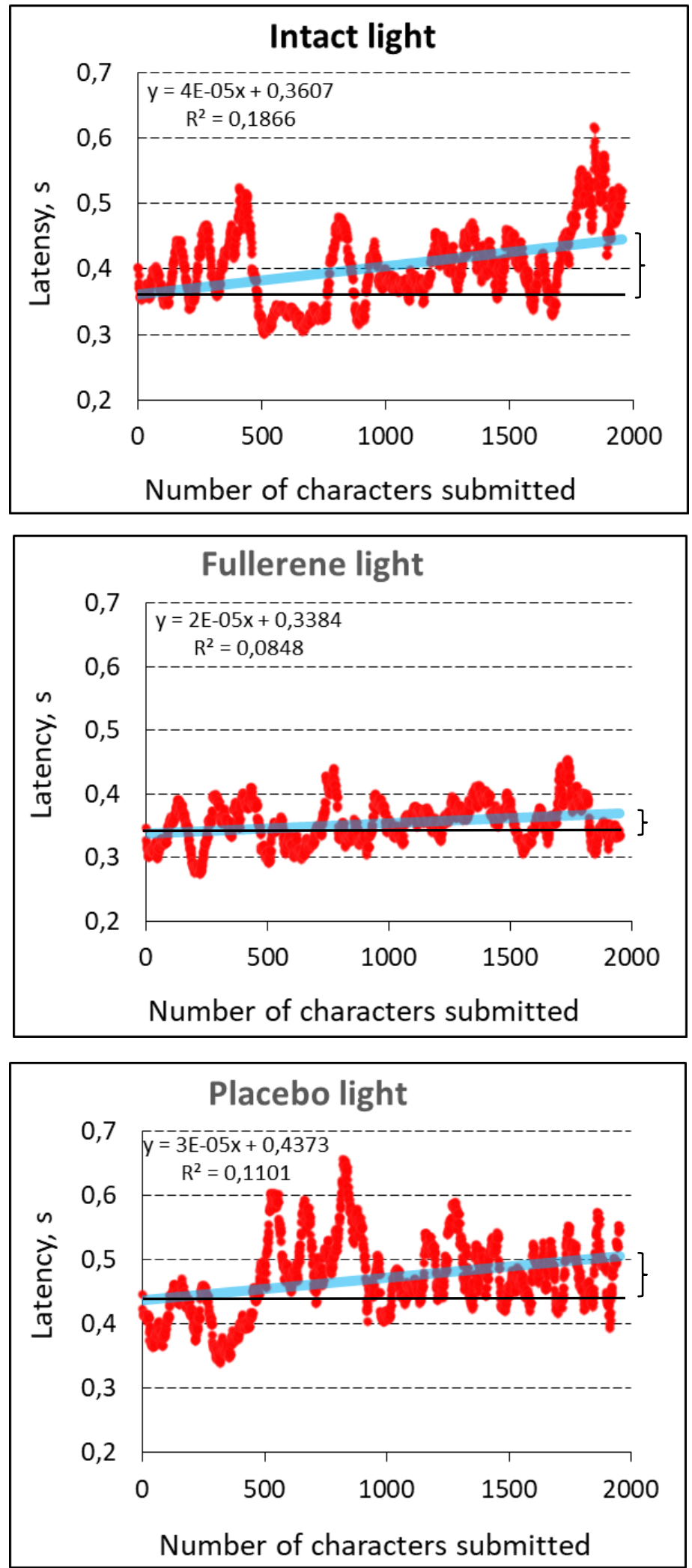

Fig. 7 Averaged for 50 consecutive symbols, the values of the latent period of the reaction of the same participant when performing a simple correction test under the influence of the studied variants of lighting the eyes. Fatigue is indicated by a bracket on the right. 
less pronounced than in intact or placebo light. If we average the latent reaction period for 50 successively displayed symbols (Fig. 7), a less pronounced deviation of the trend line from the horizontal was clearly manifested in the case of the test with fullerene light, which indicated less fatigue. For this participant, by the end of testing, fatigue was $18 \%$, whereas in intact conditions it was $66 \%$, and with placebo light it was $29 \%$.

For all participants, by the end of testing, the speed of information processing decreased, as well as the concentration ratio of task processing (Fig. 8). Both of these indicators testified to fatigue and reduced mental performance.

Fig. 8 shows that already at the very beginning of testing, the speed of information processing under the action of fullerene light was higher (1.05 bit/s) than in intact conditions (0.97) or under placebo light (0.98). This pattern also occurred when processing the last 500 characters $(0.95,0.87$ and $0.68 \mathrm{bps})$. Thus, fullerene light contributed to an increase in the speed of information processing in the conditions of mental stress.
The fullerene light had an even more noticeable positive effect on attention and concentration. During the execution of a long correction test, the concentration ratio of all participants decreased. Without glasses at the beginning of testing (the first 500 characters), the concentration ratio was $79.2 \%$, and by the end of the test (the last 500 characters) $-55.6 \%$. The gradient was $23.6 \%$. In fullerene light conditions, concentration decreased by the end of testing only by $16.2 \%$. The placebo light had no such effect, the concentration ratio here reduced by $28.5 \%$. Consequently, fullerene light contributed to a longer retention of concentration, and therefore less fatigue during testing, simulating a long work with the computer.

An increase in the efficiency of information processing during the ocular action of fullerene light, apparently, occurred due to the reduction of the response time to each presented image. We found that the latent period of reaction to the appearance of a symbol averaged $0.365 \pm 0.183 \mathrm{~s}$ when tested with fullerene light, and without glasses, $0.377 \pm 0.183 \mathrm{~s}$.

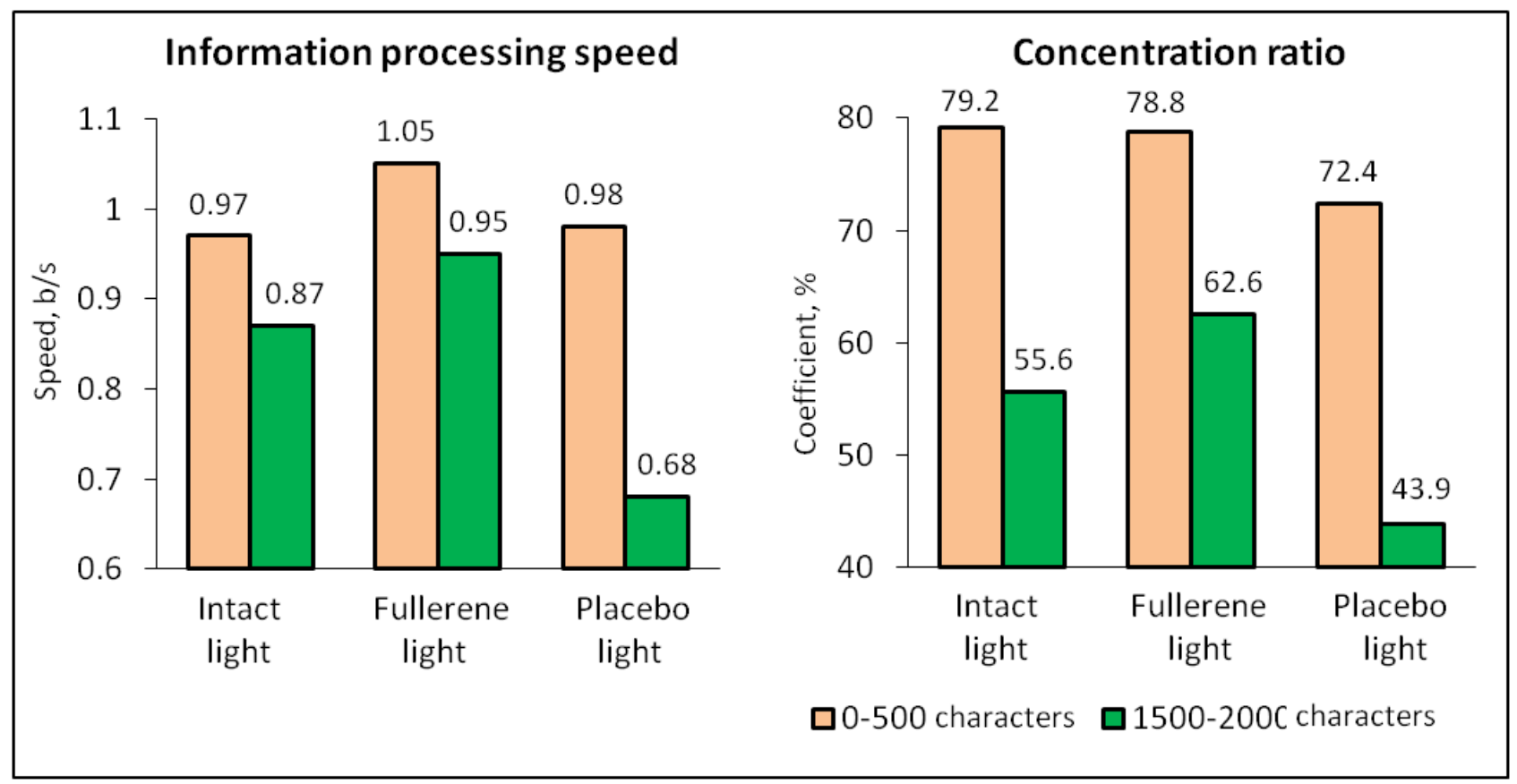

Fig. 8 Comparison of information processing speed (bit/s) and concentration ratio (\%) for a participant at the beginning of testing (reaction to the first 500 characters) and at the end of testing (reaction to the last 500 characters) when performing a simple correction test under the influence of the studied lighting options on the eye. 


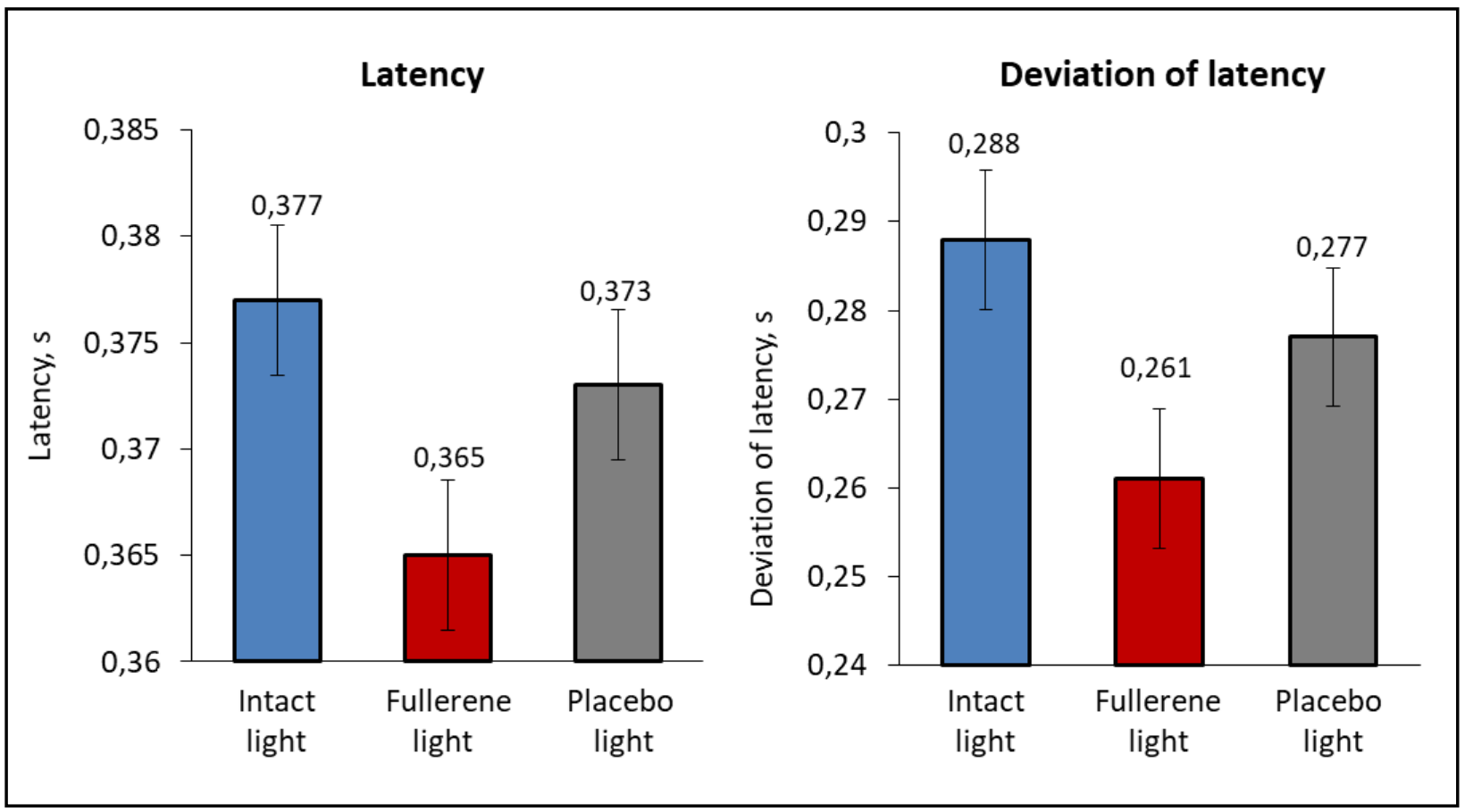

Fig. 9 The latent period and deviation of the latent period of the reaction to the appearance of the symbol in the participants (n $=10$ ) when performing a simple correction test with the ocular action of different types of light.

The mean values \pm mean deviations are indicated.

Placebo light was less effective $(0.373 \pm 0.174 \mathrm{~s})$, but more than in the control group. The latent period deviation under the action of fullerene light was the smallest (Fig. 9).

So, a simple correction test with presentation of 2,000 characters showed that when it was performed under the action of fullerene light, fatigue was significantly reduced compared with intact testing or with placebo lighting. The speed of information processing and the percentage of concentration in $\mathrm{THE}^{\circledR}$-glasses were higher, and the latent period of the reaction was shorter than with intact or placebo-light. All this testified to the fact that under the action of fullerene light on the visual system, the efficiency of information processing in the central nervous system increased.

\subsection{Correction Test of Increased Complexity for} Fatigue

Nine (9) participants passed the testing. The test required the participant to mark the rings appearing on the screen with a given orientation of the gap ("correct rings") as quickly as possible with the left hand. Of the 2,000 rings, the "correct" ones were about $10 \%$ (the interval between them was random). Along with this, rings with a different orientation of the gap ("incorrect") appeared on the screen in different sequence in time. These rings were required to mark with the right hand. Analysis of the average latent periods of reaction to the appearance of one of the 2,000 rings is shown in Fig.10.

As can be seen in Fig. 10, the response to characters with any orientation of a half-break ("wrong rings") has always been faster than to characters with a preassigned orientation of the half-break ("correct rings"). However, in both cases, under the action of fullerene light, the latent period of the reaction shortened more noticeably compared to intact lighting. Placebo light was less effective and this testified to the positive role of fullerene light in increasing the reaction rate of a person in case of intense mental stress.

The calculated average physiological latent period of the participant's reaction to the appearance of one ring in intact light was $0.39 \mathrm{~s}$, in fullerene light $-0.36 \mathrm{~s}$, and 


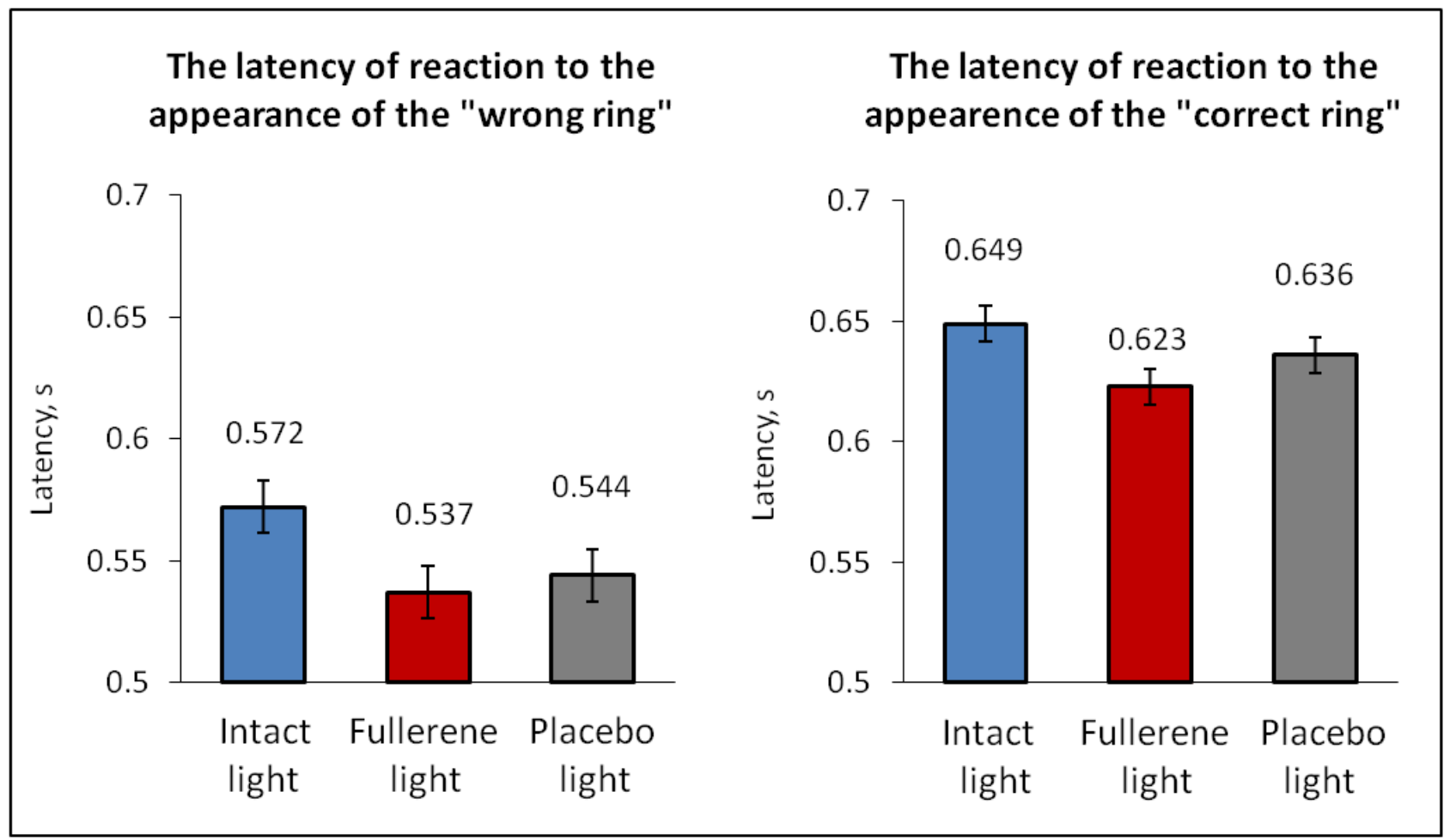

Fig. 10 The latent period of reaction (c) to the appearance of a symbol $(n=8)$ when performing a correction test of increased complexity with the ocular action of different types of light.

The mean values \pm mean deviations are indicated.

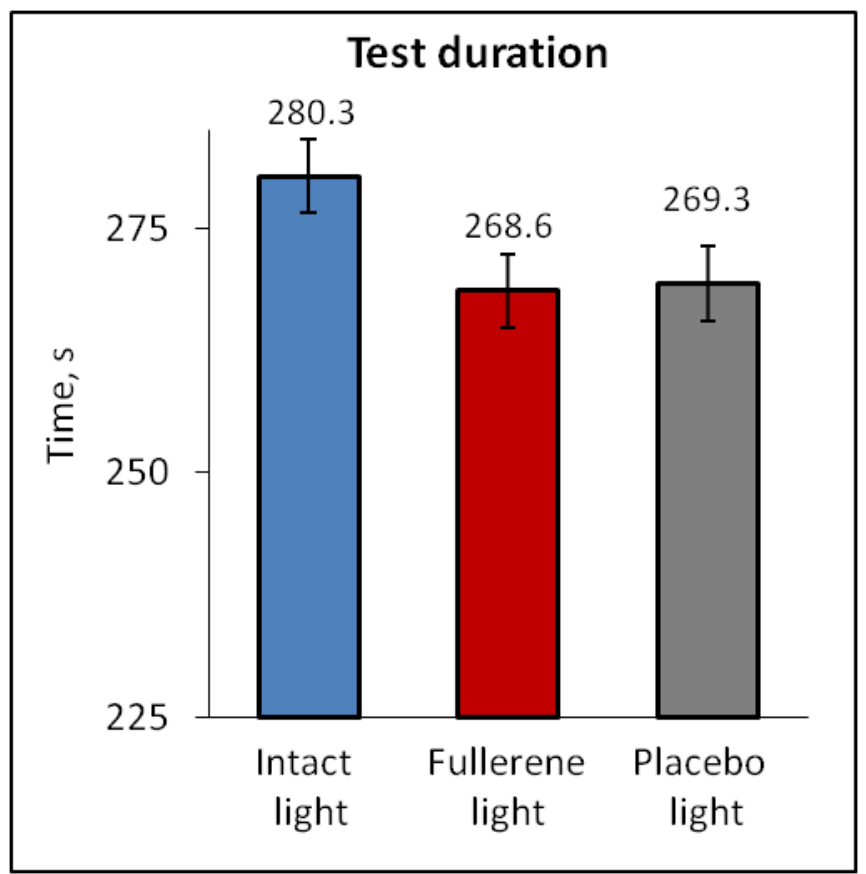

Fig. 11 Comparison of the duration of the correction test of increased complexity $(n=9)$, with the ocular action of intact, fullerene and placebo light.

The mean values \pm mean deviations are indicated. 
in placebo light $-0.37 \mathrm{~s}$. Since the latent period of the reaction to the appearance of a symbol on the screen shortened under the action of the fullerene light, it is natural that the total time spent on the test was reduced (Fig. 11). With intact illumination, the test was performed on average for $280.3 \mathrm{~s}$, and with fullerene light for $268.6 \mathrm{~s}$.

\section{Discussion}

So, when performing a correction test of increased complexity, it was found that under the action of fullerene light and placebo light there was a reduction in the latent reaction time, both on the "correct" one and on any of the 2,000 displayed symbols. The total time spent on the test, also decreased, indicating a positive (facilitating) effect of fullerene light on the visual-brain processes. This study was the first to show that the impact on the visual analyzer by the light converted by a fullerene filter, which has a positive effect on the mental performance of a person. It is implemented through increasing the efficiency of information processing by optimizing the speed and quality of performing visual-motor tasks that simulate short-term computer work [22]. To assess the performance, two tests of digital sorting (black-white and color) and two correction tests (simple and more complex) were used, imitating long-term work on a computer. All 4 tests showed that fullerene light facilitated their fulfillment.

It should be noted that experiments with human participation are always distinguished by a high spread of data, even within the same individual, since each person has constant personal concerns, the dynamic influence of social factors, different responsibilities within the test, as well as the influence of the tests themselves (substantial mental workload). Motivation can also be different for each participant. As a result, not all of the calculated parameters showed a clear effect from the fullerene light application. Thus, when performing a simple correction test, there were no statistically significant differences between the number of errors (in points), between the average values of the time of fixing attention on one ring and the net time of processing symbols. In contrast to the simple correction test, the correction test of increased complexity did not show a significant difference in the speed of information processing and a decrease in the task processing concentration by the end of the test. This distinction has no explanation as yet.

In these studies, it has identified and proven the qualitative and in some cases, quantitative positive effects of fullerene light on the indicators characterizing human sensori-motor activity. It was revealed a rapid response, which develops after the first 10-minute adaptation to fullerene light. Such pre-exposure before performing the black-white digital test shortened its duration compared to control (intact light) and placebo light. A color digital test was also performed in a shorter time than with intact light. The correction test for fatigue against the background of intact light was performed on average longer than with the use of fullerene light.

Against the background of the fullerene light, the latent period of reaction to the appearance of a ring of a preassigned configuration was shortened. For placebo light, less efficacy was observed. The physiological response time to the appearance of a single ring under the action of fullerene light was shorter than in the intact and placebo series.

The revealed increase in the efficiency of information processing during application of the fullerene light, apparently, occurs due to the reduction of the response time to each presented symbol. The average value of the speed (time) of switching attention from one symbol to another during the correction work in $\mathrm{THE}^{\circledR}$-glasses was significantly less than with intact lighting. There was also a higher rate of information processing in visual-motor structures with fullerene light, in comparison with the data obtained with intact or placebo light. Evaluation of attentiveness showed that in case of fullerene light application, significantly less error were done than in the case of performing the 
correction test in intact conditions. High quality of attentiveness was an additional confirmation of the effectiveness of mental performance when using fullerene light.

Reduction of fatigue when performing correction work, simulating long-term work on a computer, is confirmed by data obtained when comparing fullerene light, placebo light and the absence of effects (intact light). At the same time, the concentration ratio obtained at the end of the test, when the subjective signs of fatigue were already noticeable, decreased less noticeably under the action of fullerene light than under the action of placebo or intact light.

The mechanisms of influence of hyperpolarized and hyperharmonized light at different levels of biological structures have not yet been studied. We did not set a goal to study precisely the neurophysiological mechanisms of the observed effects. Their biophysical base and mechanism of transport of light signals transformed by fullerene into deep structures have not yet been determined. We believe that here plays the role, on one hand, the positive influence of fullerene light on the neurons of the central nervous system, which are involved in the implementation of visual-motor reactions, on the other hand, weakening of the harmful effects of a computer by fullerene (shortwave component). It is possible that the material (PMMA) in which low-concentration fullerene is impregnated may have some influence, although more research is needed here.

Electroencephalographic studies of humans show a positive effect on CNS neurons, involved in the implementation of visual-motor reactions [2, 3, 12, 23, 24]. It was shown that with the ocular action of fullerene light, significant differences were observed in the delta and theta ranges, which indicated changes in the state of the default networks. We revealed reduced EEG activity in the alpha range in the right frontal zone, increased EEG activity in the delta range in the temporal and posterior temporal zones, and increased EEG activity in the theta range in the posterior singular cortex. These data indicate the development of a more contemplative attitude to the introspective emotional experiences of past events with their active involvement in figurative modeling of a hypothetical future $[2,12]$.

Modeling of the driver's "blinding" according to sensorimotor reactions on the background of the EEG of the human brain we revealed an increase in the rate of interhemispheric information processes and an increase in the quality and effectiveness of decisions made [22]. It is proved a significant increase in the activity of neurons located in the posterior singular cortex, in theta and alpha ranges. This indicates improved coordination of visual information, attention, assessments of the position of the body, inhibition of irrelevant information and optimization of decision-making processes. The result of the identified changes was an increase in the speed of central information processing and a shift in the focus from image processing to decision-making processes.

These data are consistent with the results of these studies, obtained when performing visual-motor tests by humans. As was experimentally shown above, the effect on the visual analyzer of the fullerene-light has a positive effect on the mental performance of a person.

The adverse effect of radiation from computer displays is well known to specialists in the field of eyesight correction, in particular, that certain ranges of the blue part of the spectrum are most harmful to the human eye. The essence of the name "blue light" is simplified because it covers light waves, starting from the violet range (from 380 to $420 \mathrm{~nm}$ ) and to the actual blue (from 420 to $500 \mathrm{~nm}$ ). Blue light is the shortest wavelength range of visible radiation, which has the highest energy. Blue light for $40 \%$ more, compared to natural sunlight is emitted by screens of televisions, computers, pads and smartphones, thus the image on the screens seems clearer and brighter. The results of many years of research prove that prolonged exposure to the blue light affects the health of the eyes, circadian rhythms, and also provokes a number of serious 
diseases. As we have previously shown by spectrographic data [2], fullerene-containing filters contained in $\mathrm{THE}^{\circledR}$-glasses weaken the high-energy part of the solar spectrum in the UV, violet, blue, green, and yellow ranges [2, 8, 24].

When interviewing 18 participants in these studies regarding the subjective comfort of working with glasses, 16 of them noted that $\mathrm{THE}^{\circledR}$-glasses improve image sharpness, they provided less tired eyes. Two could not say if they feel better: with glasses or without glasses. This subjective opinion is confirmed by our objective results.

\section{Conclusions}

In studies on volunteers of different age groups, it was first established that the light converted by the filter containing $0.33 \%$ fullerene increases the efficiency of performing human visual-motor work (computer tests). It has been proven the positive effect of unpolarized fullerene light on a number of indicators of higher nervous activity.

When the eyesight organ was exposed to the light modified with fullerene, all the four tests used in the studies (black-white and red-black digital tests, simple and complicated correction tests) were performed in a shorter time and with fewer errors than in intact or placebo light conditions. The latent period of reaction to the appearance of a new symbol and the time of switching from one symbol to another significantly reduced. A decrease in fatigue when performing correction work, simulating long-term work on a computer, also indicates a positive effect of fullerene light on human performance. The concentration ratio, registered at the end of a lengthy correction test, when the subjective signs of fatigue were already noticeable, with fullerene-light it decreased less noticeably than under the action of placebo or intact light.

Summarizing of the obtained data suggests that the light passing through the fullerene optics/filter has a positive effect on the mental performance of a person by increasing the efficiency of information processing by the visual analyzer and creating better conditions for motor response reactions.

\section{Acknowledgments}

This work became possible due to support of Philip Zepter, President of the Zepter International, and Vibor Mulič, Vice President of the Zepter International.

\section{References}

[1] Curl, R. F., Kroto, H., and Smalley, R. E. 1996. “The Nobel Prize in Chemistry 1996." Nobelprize. org. Nobel Media AB 2014. Web. Accessed August 31, 2017. http://www.nobelprize.org/nobel_prizes/chemistry/laureat es/1996/.

[2] Gulyar, S. A., Filimonova, N. B., Makarchuk, M. Y., and Kryvdiuk, Y. N. 2019. "Ocular Influenceof Nano-modified Fullerene Light: 1. Activity of Default Networks of the Human Brain." Journal of US-China Medical Science 16 (2): 45-54.

[3] Gulyar S. A., Filimonova, N. B., Makarchuk, M. Y., and Krivdiuk, Y. N. 2019. "Ocular Influence of Nano-modified Fullerene Light: 2. Time Correlation of the Choice and Simple Sensorimotor Reactions That Determine Blinding Compensation of the Driver." Journal of US-China Medical Science 16 (3): 1-11.

[4] Koruga, D., Hameroff, S., Loutfy, R., Withers, J., and Sundereshan, M. 1993. Fullerene $C_{60}$ : History, Physics, Nanobiology, Nanotechnology. Amsterdam (North Holland): Elsevier, 1-381.

[5] Koruga, D., Simic-Krstic, J., Trifunovic, M., Jankovic, S., Hameroff, S., Withers, J., and Loutfy, R. 1993."Imaging Fullerene $\mathrm{C}_{60}$ with Atomic Resolution Using Scanning Tunneling Microscope." Fullerene Science and Technology1 (1): 93-100.

[6] Koruga, D., Matija, L., Misic, N., and Rakin, P. 1996. "Fullerene $\mathrm{C}_{60}$ : Properties and Possible Applications." Materials Science Forum 214: 49-56.

[7] Koruga, D., Nikolić, A., Mihajlović, S., and Matija, L. 2005. "Nanomagnetic Behaviour of Fullerene Thin Films in the Earth Magnetic Field in Dark and under Light Polarization Influence." Journal Nanoscience Nanotechology 5: 1660-4.

[8] Koruga, D. 2017. Hyperpolarized Light: Fundamentals of Nano Medical Photonics. Belgrade: Zepter World Book, 1-306. (in Serbian and in English)

[9] Koruga, D. 2016. "Nanophotonic Filter Based on $\mathrm{C}_{60}$ for Hyperpolarized Light." Int. and European Pat. App. PCT/EP2016/063174. Applicant: Field point, ZEPTER GROUP filed June 09, 2016 and issued October 28, 2016. 
[10] Gulyar, S. A., and Tamarova, Z. A. 2017. "Modification of Polychromatic Linear Polarized Light by Nanophotonic Fullerene and Graphene Filter Creates a New Therapeutic Opportunities." Journal of US-China Medical Science 14 (5): 173-91.

[11] Gulyar, S. A., and Tamarova, Z. A. 2018. "Influence of Many-Month Exposure to Light with Shifted Wave Range and Partial Fullerene Hyperpolarization on the State of Elderly Mice." Journal of US-China Medical Science 15 (1): 16-25.

[12] Filimonova, N. B., Makarchuk, N. E., and Gulyar, S. A. 2017. "Influence of Short-Term Ocular Exposition of Fullerene Light on the Activity of Default Chains of the Human Brain." In Proc. of the XLVII International Scientific and Practical Conference, 118-20.

[13] Gulyar, S. A., Syrota, S. S., Kiklevich, Y. N., and Pevny, S. A. 1972. "Research of Some Indicators of the Higher Nervous Activity of a Person during Long-Time Presence in the Water Environment." Fiziol Journal 18 (6): 744-50. (in Ukrainian)

[14] Gulyar, S. A., and Syrota, S. S. 1974. "The State of the Higher Nervous Activity of a Person during Prolonged Stay in a Limited Space under Pressure 3 and 5 ata." Fiziol Journal 20 (4): 440-8. (in Ukrainian)

[15] Gulyar, S. A., Moiseenko, E. V., Syrota, S., Grynevich, V. A., and Skudin, V. K. 1979. "The Effect of a Person's Being in a Nitrogen-Oxygen Environment under a Pressure of $5-12 \mathrm{kgf} / \mathrm{cm}^{2}$ on Some Indicators of Higher Nervous Activity." Fiziol Journal 25 (5): 576-84. (in Russian)

[16] Zagryadsky, V. P., and Sulimo-Samujlo, Z. K. 1978. Research Methods in the Physiology of Labor. Leningrad: Nauka, 1-93. (in Russian)

[17] Rymsky, S., and Rymskaya, R. R. 1995. Almanac of Psychological Tests, Moskow: KSP+, 1-400. (in Russian)
[18] Gulyar, S. A. 2019. "Resolved and Unresolved Issues of the Fullerene Light Therapy." In Proc. of the 50th Internat, Sci-Pract. Conf. "Application of Lasers in Medicine and Biology”, May, 22-25, 2019, Kharkiv, 158-64.

[19] Gulyar, S. A., Tamarova, Z. A., Kirilenko, Y. K., Filimonova, N. B., Makarchuk, N. Y., and Krivdiuk, Y. N. 2019. "Physiological Effects of Light with Spatial Structure Converting by Fullerene." Medical Informatics and Engineering 1 (45): 39-58.

[20] Genkin, A. A., Medvedev, V. I., and Shek, M. P. 1963. "Some Principles of the Correction Tables Construction for Determining the Speed of Information Processing." Psychology Issues 1: 104-7. (in Russian)

[21] Sysoev, V. N. 2003. Landolt E. Test. Workability Diagnostics. S-Petersburg: IMATON, 1-31. (in Russian)

[22] Gulyar, S. A., Tamarova, Z. A., and Kirilenko, E. K. 2018. "Peculiarities of Human Mental Workability under the Oculary Influence of Light Transformed by Nanophotonic Filter." In Proc. of the XLIX International Scientific and Practical Conference and 22nd Gamaleya's Readings, Oct. 3-7, 2018, Hajduszoboszlo, 59-62.

[23] Filimonova, N. B., Makarchuk, N. E., Gulyar, S. A., and Kryvdiuk, Y. N. 2018. "Influence of Light, Modified by Fulleren, on the Speed of the Brain Interhemispheric Information Processes on the Background of Changed Brightness of the Light Stimulus." In Proc. of the XLIX International Scientific and Practical Conference and 22nd Gamaleya's Readings, Oct. 3-7, 2018. Hajduszoboszlo, 163-7.

[24] Gulyar, S. A. 2018. "From Polarization to Hyperpolarization: New Aspects of Light Therapy." In Proceeding of the XLIX International Scientific and Practical Conference Proceedings of Academic Science and 22nd Gamaleya's Readings, Oct. 3-7, 2018. Hajduszoboszlo, 157-62. 